\title{
NUMERICAL STUDY ON THE INNER FLOW FIELD OF PIPES WITH VARYING CROSS-SECTIONS
}

\author{
YANHUI JIA \& ZIXUE GUO \\ Research Institute of Physical and Chemical Engineering of Nuclear Industry, P. R. China
}

\begin{abstract}
To investigate the variation regularity of refined flowing structure and flowing parameters of the working gas in pipe with varying cross-section, we numerically simulate the inner flow in the pipe using AUSM-up spatial model and LU-SGS implicit timing model, and study the influence of the Mach number on the flowing parameters. The conclusions are as follows: the structure of the inner flow is rather complicated that includes both shock wave and rarefaction wave, and there exists the overlapping phenomenon of the shock wave and the rarefaction wave, as well as the reflection of the rarefaction wave on the inner pipe wall. As the Mach number increases, the pressure at the outlet decreases whereas the temperature at the outlet ascends continuously.

Keywords: AUSM model, LU-SGS model, varying cross-section, inner flow field, numerical simulation.

\section{INTRODUCTION}

In this work, we study the inner flow in pipe with varying cross-section, the fine structure and distributing form of the flow when the gas entering the pipe, and the variation regularity of the flowing parameters including pressure, intensity, temperature and flow rate. We numerically simulate the inner flow in the pipe using AUSM-up spatial model and LU-SGS implicit timing model, and study the influence of the Mach number on the flowing parameters.
\end{abstract}

\section{CALCULATION MODEL AND METHOD}

\subsection{Physical model and meshing}

This work focuses on the 2D inner flow field in pipe with varying cross-section. The pipe is sealed with one inlet and one outlet for gas. The physical model is shown in Fig. 1.

To investigate the variation regularity of the gas flow parameters in the pipe, the physical pipe region should be converted to calculating region required for computational fluid dynamics (CFD), indicating that grid meshing is required. We take the algebraic varying method [1] to generate grids for the physical model. In this work, the calculating region is meshed by 301X71, with the total girds of 21371.

\subsection{Calculating method}

Finite volume method (FVM) conducts numerical simulation on finite controlling volumes, therefore the control equation for the gas flow in the pipe is Navier-Stokes equations in its integral form. On one controlling volume in the pipe, the integral N-S equation is:

$$
\iint_{\Omega} \frac{\partial Q}{\partial t} d \Omega+\frac{1}{\Omega_{I J}} \oiint_{\partial \Omega} \vec{F} \cdot \vec{n} d s-\frac{1}{\Omega_{I J}} \oiint_{\partial \Omega} \vec{F}_{V} \cdot \vec{n} d S=0
$$




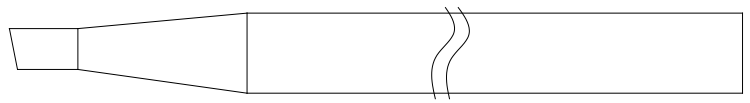

Figure 1: The schematic of the physical model of the pipe with varying cross-section.

The first, second and third terms on the left are the time derivative, non-viscous flux term and the viscous flux term, respectively. The three numerical discretization methods are different from each other, which are introduced as follows.

\subsubsection{Discretization for non-viscous flux term}

The expression for the non-viscous flux term is:

$$
\frac{1}{\Omega_{I J}} \oiint_{\partial \Omega} \vec{F} \cdot \vec{n} d s=\frac{1}{\Omega_{I J}}\left(H_{I+1 / 2, J}-H_{I-1 / 2, J}+H_{I, J+1 / 2}-H_{I, J-1 / 2}\right),
$$

where $\Omega_{I J}$ is the area of the controlling volume enclosing the central point (I, J). is:

The equation for the non-viscous flux passing the right boundary of the controlling volume

$$
\begin{gathered}
H_{I+1 / 2, J}=\left[n_{1} F_{1}+n_{2} F_{2}\right]_{I+1 / 2, J} \Delta s_{I+1 / 2, J} \\
F_{1}=\left[\begin{array}{l}
\rho u \\
\rho u^{2}+p \\
\rho u v \\
(E+p) u
\end{array}\right] \quad F_{2}=\left[\begin{array}{l}
\rho v \\
\rho u v \\
\rho v^{2}+p \\
(E+p) v
\end{array}\right],
\end{gathered}
$$

$\vec{n}=\left(n_{1}, n_{2}\right)^{T}$ is the normal direction of the right boundary, while $\Delta s_{I+1 / 2, J}$ is the length for this boundary. The flux calculating method for the other boundaries is the same as that for the right boundary.

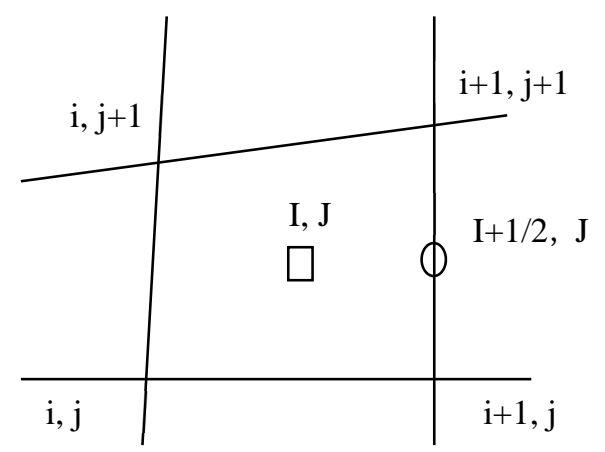

Figure 2: Schematic for the nods of the controlling volume. 
The key point for calculating the non-viscous flux is to calculate the flux $F_{I+1 / 2, J}$ at the point $(I+1 / 2$, J). In the calculation for supersonic velocity flow field, upwind format is generally used. In this work, we use $\mathrm{AUSM}^{+}$-up format, with the detailed calculating process shown in reference [2].

2.2.2 Discretization for viscous flux term

The expression for the viscous flux term is:

$$
\frac{1}{\Omega_{I J}} \oiint_{\partial \Omega} \vec{F}_{v} \cdot \vec{n} d s=\frac{1}{\Omega_{I J}}\left(H_{I+1 / 2, J}^{v}-H_{I-1 / 2, J}^{v}+H_{I, J+1 / 2}^{v}-H_{I, J-1 / 2}^{v}\right)
$$

The equation for the viscous flux passing the right boundary of the controlling volume is:

$$
\begin{aligned}
& H_{I+1 / 2, J}^{v}=\left[n_{1} F^{v}{ }_{1}+n_{2} F^{v}\right]_{I+1 / 2, J} \Delta s_{I+1 / 2, J} \\
& F_{1}^{v}=\left[\begin{array}{l}
0 \\
\tau_{11} \\
\tau_{12} \\
k \frac{\partial T}{\partial x}+u \tau_{11}+v \tau_{12}
\end{array}\right] ; \quad F_{2}^{v}=\left[\begin{array}{l}
0 \\
\tau_{12} \\
\tau_{21} \\
k \frac{\partial T}{\partial y}+u \tau_{12}+v \tau_{21}
\end{array}\right] \\
& \tau_{i j}=\frac{\mu}{\operatorname{Re}}\left(\frac{\partial u_{i}}{\partial x_{j}}+\frac{\partial u_{j}}{\partial x_{i}}-\frac{2}{3} \frac{\partial u_{k}}{x_{k}} \delta_{i j}\right) \\
& k=\frac{\mu C_{p}}{\operatorname{Pr} \operatorname{Re}}
\end{aligned}
$$

The key point for calculating the viscous flux is to calculate the derivative $\partial u_{i} / \partial x_{j}, \partial T / \partial x_{j}$ at the point $(I+1 / 2, J)$. Then, the viscous flux passing the right boundary can be calculated, the same for the viscous flux on the other three boundaries.

\subsubsection{Time format}

The flow field for specialized machines usually falls into the category of steady state problems; therefore, the time format should perform in the implicit format that has the fast convergence speed. LU-SGS (Lower-Upper Symmetric Gauss-Seidel) is utilized as the calculating method; the detailed process for deducing this method is shown in reference [3].

\subsection{Calculating conditions}

2.3.1 The free inner flow condition and initial condition

The known parameters for the flow are: characteristic length $L_{\text {ref }}$, inward flow temperature $T_{\infty}$, inward flow density $\rho_{\infty}$, inward flow Mach number $M a_{\infty}$, inward flow pressure $p_{\infty}$, inward flow viscosity $\mu_{\infty}$, inward flow Reynolds number $\mathrm{Re}_{\infty}$, and inward flow angle $A o A$. 


\subsubsection{Boundary conditions}

The boundary condition for this model compromises of three conditions, which are the inlet condition, outlet condition and wall condition. At the inlet, the condition is the supersonic inlet condition. The physical parameters on the boundary are determined solely by the inward flow. At the outlet, the condition is the supersonic outlet condition; all the physical parameters are deduced by the inner nods on the grids. The wall is non-slip, therefore the gas rate at the wall is zero. The normal component of the gradient of the gas pressure is zero. The temperature on the wall is determined by the equal temperature wall or the thermally isolated wall.

\section{DISCUSSION}

\subsection{Analysis of the inward flow in pipe}

By using the numerical method mentioned, we calculated the flow structure of the working gas in the pipe. The distribution of gas pressure, density, temperature and Mach number are shown in Figs 3-6. The results indicate that the structure of the inner flow is rather complicated that includes both shock wave and rarefaction wave, and there exists the overlapping phenomenon of the shock wave and the rarefaction wave, as well as the reflection of the rarefaction wave on the inner pipe wall.

The airflow at the front end of the inner wall of the pipe forms a slanting wave due to the formation of a compression deflection angle between the free flow and the upper wall. Due to the high intensity of oblique shock, the physical quantities (pressure, density, temperature and Mach number, etc.) of the airflow before and after the oblique wave are greatly changed. According to the distribution of physical quantities, the pressure increases by 8.1 times, the density increases by 6.5 times, the temperature increases by 1.3 times, and the Mach number decreases by $22 \%$. The airflow at the front end of the inner wall of the pipe forms a bunch of sparse waves, and the airflow is in the process of decompression and expansion. According to the distribution of the physical quantities, it can be seen that the pressure of the airflow is about $73 \%$ lower than that of the free flow after the expansion of the sparse wave. The gas density also decreases by about $75 \%$, but the gas temperature increases by 1.4 times.

As the airflow continues to flow along the pipeline, the oblique and sparse expansions of the ends intersect. The process of the two airflow parameters before and after the intersection is roughly as follows: When the shock wave intersects the sparse beam, the shock wave is bent at the intersection and becomes the curve shock, which is due to the mismatch between the shock wave and the boundary condition of the airflow after the sparse beam (Speed, velocity direction, pressure magnitude, etc.). After the intersection, the air flow in order to re-reach the matching state (after the intersection of air parameters must be consistent), it will produce a shock wave and a wave of expansion continue to flow along the pipeline.

After the intersection of the airflow, the newly generated shock wave encounters the lower wall (solid wall) of the pipeline during the movement, and the steady phenomenon of the shock wave and the solid wall surface occurs. From the physical distribution cloud diagram, the shock wave (hereinafter referred to as "reflected shock"), but the reflection angle is less than the angle of incidence, that is, after deflecting the direction of the air flow velocity deflected by a certain angle, tend to re-with the pipe wall Parallel face. From the pressure distribution cloud, we can see that this shock wave and the wall of the pipeline multiple reflection deflection, but the reflection intensity gradually weakened, the direction of air flow continuously parallel to the direction of the wall deflection. 
Figure 3: Distribution of the gas pressure in the pipe.

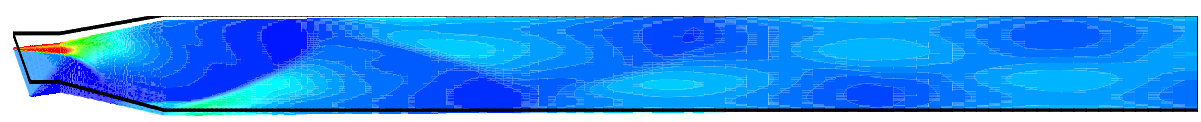

Figure 4: Distribution of the gas density in the pipe.

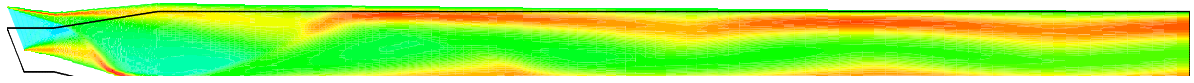

Figure 5: Distribution of the gas temperature in the pipe.

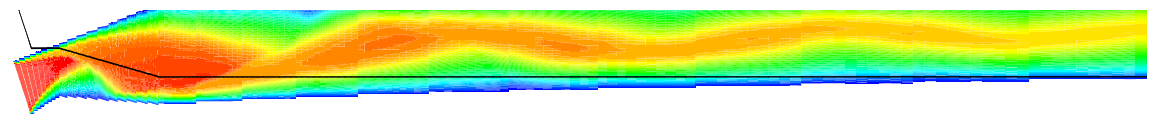

Figure 6: Distribution of the gas Mach number in the pipe.

From the temperature contour cloud (Fig. 5), the temperature of the airflow in the pipe is significantly higher than that of the free flow temperature, except for the small triangular region before the intersection of the head shock and the expansion wave. At the same time, the temperature near the upper and lower wall of the pipeline is high, and it is concluded that this is the result of the double heat of the viscous boundary layer on the wall and the reflection of the wall shock.

It is known that the supersonic flow $(\mathrm{Ma}>1)$ and subsonic velocity $(\mathrm{Ma}<1)$ coexist in the inner flow field, but there are obvious differences in the mechanism of the two. The subsonic flow region appears near the stationary tube wall due to the viscous boundary layer produced by the friction between the solid wall and the viscous gas. The gas velocity should be zero on the solid wall surface and the velocity gradient in the boundary layer is large, so in the distance from the wall of the air flow (supersonic flow) under the action of the gas velocity rapidly increased to supersonic. The characteristics of the supersonic flow region are consistent with the flow structure of the previous analysis. With the intersection and reflection of the shock and the expansive wave, the airflow parameters are constantly changing accordingly, and the final velocity of the air flow is still supersonic.

\subsection{The Mach number of the inward flow}

This paper simulates the change rule of the pipeline flow when the Mach number is 0.9 and 1.1 respectively. The calculated results show that the distribution of gas flow in the pipeline s not very different when the number of free flows is changed; there are oblique shocks and sparse waves in the front end, followed by the intersection of the shock wave and the sparse wave and the intersection of the oblique wave and the solid wall. 


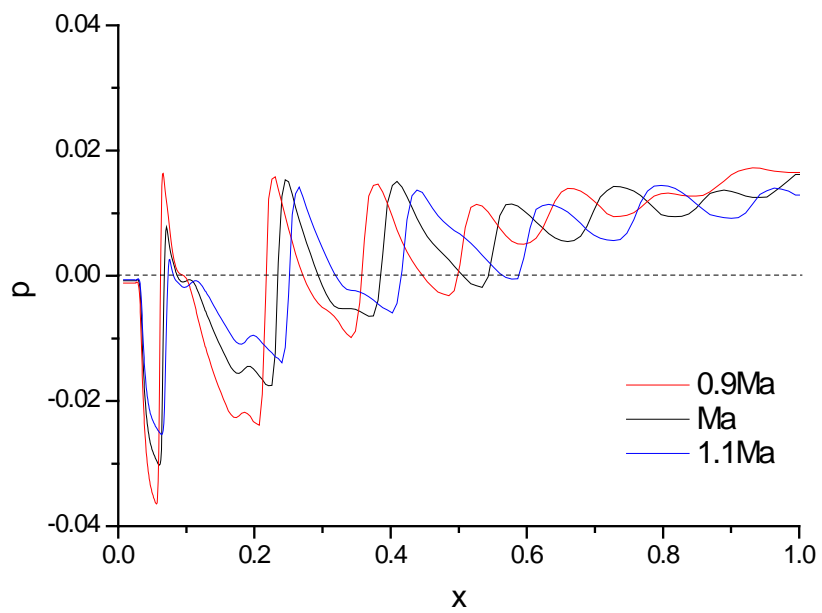

Figure 7: The pressure distribution at the pipe central line with different Mach number.

From the central axis of the cross-section of the pressure coefficient distribution curve (shown in Fig. 7) we can see that different flow Mach number of pressure coefficient distribution trends are consistent (all showed a rising trend, the gas pressure gradually in an outlet pressure value). From the export pressure coefficient is greater than zero, we can see that the outlet pressure is greater than the inlet pressure, that is, after the flow through the pipeline to achieve pressurization.

The differences are mainly concentrated in the fluctuating curve of the location of the valley and the slope of the asymptotes. The difference between the peak and valley positions indicates that the position of the shock wave reflected on the wall of the pipe is different, and as the Mach number increases, the shock reflection point moves toward the pipe outlet. The slope of the progressive line decreases when the incoming Mach number increases, indicating that the outlet pressure decreases with the increase of the Mach number of the incoming flow.

\section{CONCLUSION}

The flow structure of the variable cross-section pipe is complicated, and the shock wave and the sparse wave coexist, and there is the intersection of the shock wave and the sparse wave and the shock reflection of the pipe wall. Compared with the free flow, the air outlet speed is still supersonic, pressure increases, the temperature increases. The incoming Maher number is an important input condition for the airflow. After the Mach number increases, the oblique shock intensity of the pipeline head is obviously increased, and the subsequent shock reflection point is moved to the pipe exit. With the flow of Mach number increases, the export pressure decreases, while the export temperature continues to rise.

\section{REFERENCE}

[1] Hoffmann, K.A. \& Chiang, S.T., Computational Fluid Dynamics. USA: a Publication of Engineering Education System, 2000.

[2] Liou, M.S., A Sequel to AUSM, Part II: AUSM+-up for all Speed. Journal of Computational Physics, (214), pp. 137-170, 2006.

[3] Seokkwan Yoon, Antony Jameson. Lower-Upper Symmetric-Gauss-Seidel Method for the Euler and Navier-Stokes Equations. AIAA Journal, 26(9), pp. 1025-1026, 1988. 